\title{
LncRNA GAS5 suppresses the proliferation and invasion of osteosarcoma cells via the miR-23a- 3p/PTEN/PI3K/AKT axis
}

Jianmin Liu

Shaanxi Provincial People's Hospital

Ming Chen ( $\square$ chenwn1911@163.com )

Shaanxi Provincial People's Hospital https://orcid.org/0000-0002-2487-3514

Longyang Ma

Shaanxi Provincial People's Hospital

Xingbo Dang

Shaanxi Provincial People's Hospital

Gongliang Du

Shaanxi Provincial People's Hospital

\section{Primary research}

Keywords: Long non-coding RNA, growth arrest special 5, osteosarcoma, miR-23a-3p, phosphatase and tensin homolog

Posted Date: May 12th, 2020

DOI: https://doi.org/10.21203/rs.3.rs-26945/v1

License: (1) This work is licensed under a Creative Commons Attribution 4.0 International License.

Read Full License

Version of Record: A version of this preprint was published at Cell Transplantation on January 1st, 2020. See the published version at https://doi.org/10.1177/0963689720953093. 


\section{Abstract}

Background: Accumulating evidence has shown that IncRNA growth arrest special 5 (GAS5) is a well-known tumor suppressor in the pathogenesis of a variety of human cancers. However, the detailed role of GAS5 in osteosarcoma is largely unclear. Here, we explore the role of GAS5 in progression of osteosarcoma.

Methods: The expression level of GAS5 was detected in human osteosarcoma tissues and matched adjacent tissues, as well as osteosarcoma cell lines and nonmalignant osteoblast cells. Then, in vitro gain- and loss-of-function experiments, with the pcDNA-GAS5 expression vector and GAS5-siRNA, were performed in U2OS and HOS cells to determine the effect of GAS5 on osteosarcoma cell proliferation and invasion. Subsequently, we searched potential miRNA targets with bioinformatics analysis and confirmed their interaction by using luciferase reporter gene and RNA pull-down assays. The function and mechanism of miR-23a-3p in proliferation and invasion was also investigated in U2OS and HOS cells. Furthermore, rescue experiments were performed to verify the involvement of miR-23a-3p and its target gene in GAS5-mediated cell behaviors. Finally, a xenograft nude mouse model was established by subcutaneous injection with U2OS cells overexpressing GAS5 or not, and the effect of GAS5 on tumor growth in vivo was evaluated.

Results: GAS5 was downregulated in human osteosarcoma tissues and cell lines. Overexpression of GAS5 could significantly suppress, and downregulation of GAS5 promoted, proliferation and invasion of osteosarcoma cells. GAS5 could directly bind with and upregulated miR-23a-3p, which posttranscriptionally downregulated the tumor suppressor PTEN and positively regulated proliferation and invasion of osteosarcoma cells. Rescue experiments confirmed the involvement of miR-23a-3p and PTEN in GAS5-mediated cell behaviors by modifying the phosphatidylinositol-3-kinases/protein-serine-threonine kinase (PI3K/AKT) pathway. GAS5 could inhibit tumor growth in vivo.

Conclusion: GAS5 functions as a competing endogenous RNA, sponging miR-23a-3p, to promote PTEN expression and suppress cell growth and invasion in osteosarcoma by regulating the PI3K/AKT pathway.

\section{Background}

Osteosarcoma is the most common malignant bone tumor which most frequently occurs in young adults and children and has high metastasis and recurrence rates[1-3]. With the application of surgery combined with chemotherapy, the 5-year survival rate of patients with non-metastatic osteosarcoma is between $60 \%$ and $70 \%$, but the survival rate of patients with metastatic osteosarcoma is only $11-13 \%[4-6]$. Therefore, it is urgent to elucidate the pathogenesis and molecular mechanisms of osteosarcoma, which will be helpful for the development of effective treatments of osteosarcoma.

Long noncoding RNAs (IncRNAs) are a class of protein-non-coding transcripts with a length greater than 200 nucleotides[7, 8]. Increasing evidence has revealed the important regulatory effects of IncRNAs on a variety of physiological and pathological processes, especially cancer[8, 9]. LncRNA growth arrest- 
specific transcript 5 (GAS5) has been demonstrated to be involved in several human cancers, and acts as a tumor suppressor[10]. An in vitro investigation revealed that GAS5 could suppress pancreatic cancer metastasis[11]. In gastric cancer, overexpression GAS5 suppresses tumorigenesis and development[12]. However, the potential role of GAS5 in osteosarcoma is largely unknown.

It has been reported that IncRNAs could serve as competing endogenous RNAs (ceRNAs) by interfering with the expression of miRNAs to be involved in the occurrence and development of cancers[2,13]. As is well known, miRNAs also could bind to different mRNAs to regulate the development of multiple tumors[14, 15], including bladder cancer, pancreatic cancer, osteosarcoma and so on [16-18]. But the relationship between IncRNA and miRNA is not entirely clear yet[19]. Some research suggested that IncRNAs might sponge miRNAs to sequestering miRNAs away from their target mRNAs [2, 20, 21]. Hence, exploring the function of IncRNA-miRNA-mRNA crosstalk could be expected to become a key development in the mechanism and pathogenesis of cancers. MiR-23a-3p level was upregulated in a variety of tumors, indicating that miR-23a-3p could be involved in the occurrence and development of tumors[22]. Moreover, miR-23a-3p might promote tumor development by inhibiting the expression of phosphatase and tensin homolog (PTEN)[23]. In the view of these findings, we were interested in whether miR-23a-3p regulated the level of PTEN to be involved in the development of osteosarcoma.

In this study, we found that IncRNA GAS5 was also downregulated in human osteosarcoma tissues, compared with matched adjacent tissues. Then, we explored the role and underlying mechanisms of GAS5 in osteosarcoma cells. Bioinformatics analysis, luciferase reporter gene RNA immunoprecipitation and pull-down assay were used to determine the targeted interaction between GAS5 and miR-23a-3p. And the effect of GAS5 on miR-23a-3p/PTEN and PI3K/AKT signaling pathway was also investigated in osteosarcoma. Furthermore, in vivo xenograft experiment was used to explore the effect of GAS5 on osteosarcoma growth. This investigation may be an effective strategy for the treatment of osteosarcoma.

\section{Materials And Methods}

\section{Clinical Specimens}

Twenty osteosarcoma tissues and their matched adjacent tissues were collected from patients ( 15 males and 5 females; average age 19 years old) who received surgical treatment at the Shaanxi Provincial People's Hospital from February 2017 to November 2018. All the patients had not received preoperative radiotherapy and/or chemotherapy and were obtained with informed consent before surgery. The histological diagnosis of osteosarcoma conformed to the World Health Organization's histological criteria for osteosarcoma and the protocols were approved by the Ethics Committee of Shaanxi Provincial People's Hospital. Tissues were frozen in liquid nitrogen immediately and stored at $-80^{\circ} \mathrm{C}$.

\section{Cell Lines and Culture}

Human normal osteoblast cells (hFOB1.19) and human osteosarcoma cell lines (Saos2, 143B, MG-63, U2OS, HOS) were purchased from American Type Culture Collection (ATCC, Manassas, VA, USA). All cells 
were incubated in DMEM (Gibco, Rockville, MD) containing $10 \%$ fetal bovine serum (Gibco, Rockville, $\mathrm{MD}$ ) and $100 \mathrm{U} / \mathrm{mL}$ penicillin and $100 \mu \mathrm{g} / \mathrm{mL}$ streptomycin (Sigma, St. Louis, MO, USA) at $37^{\circ} \mathrm{C}$ in a humidified atmosphere with $5 \% \mathrm{CO}_{2}$.

Reverse Transcription-quantitative PCR (RT-qPCR)

Total RNA was extracted from tissues and cells using Trizol reagent (Invitrogen, Carlsbad, CA, USA) according to the manufacturer's instructions. RNA samples were performed using M-MLV Reverse Transcriptase (Takara Biotechnology, Dalian, China) and cDNA amplification was carried out using the SYBR Green PCR Master Mix (Applied Biosystems). PCR amplification was carried out using an Applied Biosystems $7900 \mathrm{HT}$ Fast Real-Time PCR System following the thermal cycling conditions: $95^{\circ} \mathrm{C}$ for 10 min; 40 cycles of $95^{\circ} \mathrm{C}$ for $15 \mathrm{~s}$ and $60^{\circ} \mathrm{C}$ for $60 \mathrm{~s}$. GAPDH was used as the internal control for normalizing mRNA expression. Real time quantitative polymerase chain reaction was performed using the TaqMan microRNA Reverse Transcription Kit (Applied Biosystems, Foster City, CA, USA) and following parameter values to program the thermal cycler: $16^{\circ} \mathrm{C}$ for $30 \mathrm{~min}, 42^{\circ} \mathrm{C}$ for $30 \mathrm{~min}$ and $85^{\circ} \mathrm{C}$ for $5 \mathrm{~min}$. U6 was served as the internal control for the normalization of miRNA expression. The relative expression of mRNA or miRNA was normalized and calculated by using the $2^{-\triangle \Delta C t}$ method. The following primers were used: GAS5 (forward 5'-3': ACAGGCATTAGACAGAAAGC; reverse5'-3': TACCCAAGCAAGTCATCCA); miR-23a-3p (forward 5'-3': ACACTCCAGCTGGGATCACATTGCCAGGGATTT; reverse 5'-3': CTCAACTGGTGTCGTGGAGTCGGCAATTCAGTTGAGGGAAAT CC); PTEN (forward 5'-3': ACCAGTGGCACTGTT GTTTCAC; reverse 5'-3': TTCCTCTGGTCCTGGTATGAAG).

\section{Cell Transfection}

Human GAS5 gene was amplified by PCR from hFOB1.19 cells and cloned into pcDNA3.1 vector (Sangon, Shanghai, China) to construct pcDNA-GAS5 plasmid, with the empty pcDNA3.1 vector serving as the negative control (Vector). Plasmids were transfected into HOS and U2OS cells using Lipofectamine 3000 Transfection Reagent, according to the manufacturer's instructions (Invitrogen, Carlsbad, CA, USA). MiR23a-3p mimic, inhibitor, and its corresponding negative control mimic or inhibitor (NC-mimic and NCinhibitor) were bought from Santa Cruz Biotechnology (Santa Cruz, CA, USA). In addition, siRNA negative control (Scramble), si-GAS5, and si-PTEN (All obtained from Sangon, Shanghai, China) were transfected into cells as described above.

\section{Analysis of Cell Proliferation}

Cell proliferation was detected by using CCK-8 assay. Briefly, cells were seeded into 96-well plates and cultured for $24 \mathrm{~h}$. After transfection for $0,24,48 \mathrm{~h}$ and $72 \mathrm{~h}, 10 \mu \mathrm{L}$ CCK-8 solution was added into each well and co-incubated with cells for another $2 \mathrm{~h}$ at $37^{\circ} \mathrm{C}$. The absorption values were detected at wavelength of $450 \mathrm{~nm}$ with a micro-plate analyzer (Molecular Devices, Sunnyvale, CA, USA).

Invasion Assays 
Cell invasion was determined using Matrigel ${ }^{\mathrm{TM}}$ Invasion Chambers. Briefly, $1 \times 10^{5}$ cells in $200 \mu \mathrm{L}$ freeserum DMEM medium were seeded into the upper compartment of 24-well plates, while $600 \mu \mathrm{L}$ medium, containing $20 \%$ FBS, was added to the lower chamber. After incubation for $48 \mathrm{~h}$, cells on the upper membrane surface were removed using a cotton swab and invaded cells were fixed with $70 \%$ ethanol for $10 \mathrm{~min}$ and stained with $0.1 \%$ crystal violet for $15 \mathrm{~min}$. These invasive cells that had moved across the membrane were counted from five randomly selected fields using a light microscope (magnification, 200 $\mathrm{x}$, Tokyo).

\section{Cell Apoptosis}

Flow cytometry was used to evaluate cell apoptosis. After transfection, cells were collected and washed with phosphate-buffered saline for three times. Cells were stained with $5 \mu \mathrm{L}$ Annexin V-FITC and $5 \mu \mathrm{L}$ propidium iodide at room temperature in the dark for $20 \mathrm{~min}$, cell apoptosis were examined by using flow cytometry (BD Biosciences, USA).

\section{RNA Immunoprecipitation (RIP) and RNA pull-down}

RNA immunoprecipitation (RIP) assay was performed to explore the interaction between GAS5 and miR23a-3p with EZ-Magna RIP RNA-binding protein immunoprecipitation kit (Millipore). After cell lysis with RIP lysis buffer, $100 \mu \mathrm{L}$ of the lysate was incubated with RIP immunoprecipitation buffer containing magnetic beads, which were conjugated with human anti-Argonaute2 (Ago2) antibodies (Millipore, Billerica, MA, USA) or control normal mouse immunoglobulin G (IgG; Millipore, Billerica, MA, USA). Among the antibodies, IgG was considered as a negative control. Proteinase $\mathrm{K}$ buffer was then added to the samples to digest protein. Finally, the target RNA was extracted and purified for further study by RT-qPCR.

RNA pull-down assay was performed to examine the interaction between GAS5 and miR-23a-3p. Briefly, cells were transfected with biotinylated miRNA, collected $48 \mathrm{~h}$ after transfection. The cell lysates were incubated with M-280 streptavidin magnetic beads (Invitrogen, San Diego, CA, USA). The bound RNAs were purified using TRIzol reagent (Invitrogen) for further RT-qPCR analysis.

\section{Luciferase Reporter Gene Assay}

The luciferase reporter gene assay was conducted on the basis of manufacturer's instructions. Using PCR, we amplified the putative miR-23a-3p target binding sites in

GAS5 and the GAS5 mutant binding sequence. We then subcloned into a pmirGLO Reporter plasmid (Promega, Madison, WI, USA) to construct the wild-type GAS5 3'-UTR (GAS5-WT), mutant GAS5 3'-UTR (GAS5-Mut), PTEN 3'-UTR (PTEN-WT) and mutant PTEN 3'-UTR (PTEN-Mut). Luciferase reporter plasmids and miR-23a-3p mimic or NC-mimic were co-transfected into HEK-293T cells using Lipofectamine 3000. After transfection for $48 \mathrm{~h}$, relative luciferase activity was measured by using a Dual-Luciferase Reporter Assay System (Promega, Madison, Wisconsin, WI, USA).

\section{Western blotting}


After transfection, cells were harvested and lysed in Radio Immunoprecipitation Assay (RIPA) lysis buffer (Beyotime, Shanghai, China) for $30 \mathrm{~min} 4{ }^{\circ} \mathrm{C}$. Protein concentration was quantified by using an Enhanced BCA Protein Assay Kit (Beyotime, Shanghai, China). Protein samples were separated on $10 \%$ sodium dodecyl sulfate polyacrylamide gel electrophoresis (SDS-PAGE) and transferred to polyvinylidene difluoride (PVDF) membranes (Millipore, Bedford, MA, USA). After blocked with $5 \%$ skim milk for $1 \mathrm{~h}$ at room temperature, the blots were probed with primary antibodies overnight at $4^{\circ} \mathrm{C}(\mathrm{PTEN}, 1: 2000$ dilution; PI3K, 1:0000 dilution; phosphorylated PI3K (p-PI3K), 1:0000 dilution; AKT, 1:0000 dilution; phosphorylated AKT (p-AKT) 1:2000 dilution;) (Abcam, Cambridge, UK). Then the membranes were washed and incubated with secondary antibodies (1:2000 dilution; Abcam, Cambridge, MA, USA) for $1 \mathrm{~h}$ at room temperature. Then, the chemiluminescent reagent was added to the membrane in an even manner and developed the image with a developing solution. All western blots were subjected to relative optical density (OD) analysis during the experiment. Finally, the signals were visualized using a chemiluminescence imaging system (Bioshine ChemiQ 4800 mini, China, Oxiang, Shanghai).

\section{Tumor Xenograft Assay}

All animals were approved by the Ethics Committee for the Use and Care of Animals of Shaanxi Provincial People's Hospital. Female BALB/c nude mice (age:4 weeks old; weight: 16-20 g;) were purchased from Institute of Laboratory Animals in Chinses Academy of Medical Sciences (Shanghai, China). The mice were randomly divided into two groups, and $2 \times 10^{6}$ Vector or pcDNA-GAS5 transfected U2OS cells were subcutaneously injected into the right armpit of each mice. Twenty-eight days later, the mice were sacrificed, and the cancer tissues were harvested. The tumor volume was calculated every week with the formula $\left(\mathrm{mm}^{3}\right)$ : tumor volume=length $\times$ width $^{2} \times 0.5$. The body weight of the mice was measured using electronic scale every 7 days. The level of GAS5 and miR-23a-3p in tumors was detected using RT-qPCR and the protein level of PTEN was analyzed using western blot.

\section{Statistical Analysis}

Data are reported as the mean \pm SEM and processed with SPSS version 22.0 software (IBM Corp., Armonk, NY, USA). T-test was used to compare the differences between the two groups. One-way analysis of variance (ANOVA) was used for the difference analysis of three groups and more than three groups. ** $P \otimes 0.05$ was considered to be statistically significant.

\section{Results}

LncRNA GAS5 is downregulated in osteosarcoma tissues and cell lines

To define the potential function of GAS5 in osteosarcoma, we first detected the level of GAS5 in osteosarcoma tissues and matched adjacent tissues from twenty patients with osteosarcoma by RTqPCR. And we found that the expression level of GAS5 in the osteosarcoma tissue was significantly lower 
than the adjacent tissue (Fig. 1A). Additionally, we also analyzed the expression of GAS5 in five osteosarcoma cell lines and a normal osteoblast cell line. The levels of GAS5 were dramatically downregulated in osteosarcoma cell lines Saos2, 143B, MG-63, U2OS and HOS compared with hFOB1.19 (Fig. 1B). When cells were transfected with pcDNA-GAS5, the level of GAS5 observably increased relative to control group (Fig. 1C), while dramatically decreased after transfection with si-GAS5 (Fig. 1D). These results indicated that the level of GAS5 was downregulated in osteosarcoma.

Upregulation/downregulation of GAS5 inhibits/promotes osteosarcoma proliferation and invasion through PI3K/AKT signal pathway

Since U2OS and HOS cells exhibited the lowest expression of GAS5, thus, U2OS and HOS cells were selected for further analysis. CCK-8, Transwell and Flow cytometry assays showed that overexpression of GAS5 could suppress cell proliferation viability (Fig. 2A and B), reduce the number of cell invasion (Fig. $2 \mathrm{C}$ and $\mathrm{D}$ ) and increase cell apoptosis (Fig. 2E and F), while downregulation of GAS5 could promote cell proliferation (Fig. $3 \mathrm{~A}$ and B), increase cell invasion (Fig. $3 \mathrm{C}$ and D) and inhibit cell apoptosis (Fig. 3E and F). Western blot assay showed that the phosphorylation levels of PI3K and AKT decreased after transfection with pcDNA-GAS5 (Fig. 2G) but increased after transfection with si-GAS5 (Fig. 3G). All results suggested that GAS5 was involved in the proliferation and invasion of osteosarcoma through PI3K/AKT signal pathway.

GAS5 is a direct target gene of miR-23a-3p and regulates the expression of miR-23a-3p

LncRNA could inhibit the expression and activity of miRNA[24], so we used StarBase to predict the potential miRNA binding sites in GAS5. As shown in Fig. 4A, there is a complementary binding site between GAS5 and miR-23a-3p. To validate this prediction, luciferase reporter assay was performed. The luciferase activity of WT-GAS5 was significantly reduced by miR-23a-3p mimic as compared to control in the WT-GAS5 group, but that there was no signification change in the Mut-GAS5 group (Fig. 4B). It has been proved that miRNAs exert their functions of gene silencing by recruiting nuclear localized Ago2. To conform whether GAS5 and miR-23a-3p are in the same Ago2 immunoprecipitants, RIP assay was performed. IgG was used as a negative control. The results suggested that both GAS5 and miR-23a-3p were enriched in Ago2 pellet in comparison with IgG control group (Fig. 4C). Furthermore, a biotin-avidin pulldown assay was measured to analyzed whether miR-23a-3p could pull down GAS5. The result revealed that GAS5 was pull down by miR-23a-3p, while failed to the mutated miR-23a-3p, suggesting that GAS5 could regulate miR-23a-3p in a sequence-specific manner (Fig. 4D). We also found that miR23a-3p expression was higher in osteosarcoma tissues than that in adjacent tissues (Fig. 4E). The level of miR-23a-3p significantly upregulated in osteosarcoma cell lines (Fig. 4F). Moreover, the levels of miR23a-3p were downregulated when U2OS and HOS cells were transfected with pcDNA-GAS5, while upregulated after transfection with si-GAS5 (Fig. 4G and H). These data indicated that GAS5 directly bound to miR-23a-3p and regulated the expression of miR-23a-3p.

Downregulation of miR-23a-3p inhibits cell growth and invasion through activating PI3K/AKT signal pathway 
We conducted further studies on how miR-23a-3p regulated the proliferation and invasion of osteosarcoma cells. As shown in Fig. 5A, when U2OS and HOS cells were transfected with miR-23a-3p inhibitor, the level of miR-23a-3p remarkably reduced, and the viability of cells decreased obviously compared with control group (Fig. 5B and C). Transwell assay showed that the number of cell invasion decreased after transfection with miR-23a-3p inhibitor (Fig. 5D). Cell apoptosis were determined by using flow cytometry. The results demonstrated that downregulation of miR-23a-3p could significantly promote cell apoptosis as compared to that in control group (Fig. 5E and F). Western blot experiments showed that downregulation of miR-23a-3p could reduce the phosphorylation of PI3K and AKT (Fig. 5G). These results indicated that miR-23a-3p promoted cell proliferation and invasion by activating PI3K/AKT signal pathway in osteosarcoma cells.

\section{PTEN is a target gene of miR-23a-3p in osteosarcoma cells}

By utilizing StarBase, we found that miR-23a-3p could potentially target PTEN (Fig. 6A). Luciferase assay indicated that miR-23a-3p mimic significantly inhibited the luciferase activity of in WT-PTEN group $(P<0.01)$, but not completely inhibited the luciferase activity in Mutant1 or Mutant 2 of PTEN groups. When both binding sites were mutated at the same time, luciferase activity was basically unaffected (Fig. 6B). We also analyzed mRNA and protein levels of PTEN in osteosarcoma tissue samples and the corresponding adjacent tissues. The results found that the level of PTEN in osteosarcoma tissues was significantly lower than that in adjacent tissues, suggesting that it might be involved in tumor development (Fig. 6C and D). The mRNA level of PTEN was also downregulated in osteosarcoma cell lines compared with hFOB1.19 (Fig. 6 E). Moreover, PTEN expression obviously increased in pcDNA-GAS5 and miR-23a-3p inhibitor groups, while decreased in si-GAS5 and miR-23a-3p mimic group (Fig. 6 F-G). In addition, western blot revealed that upregulation of miR-23a-3p repressed the protein level of PTEN but promoted the expression of phosphorylated PI3K and AKT (Fig. 6H). Silence of miR-23a-3p showed the opposite results. These findings revealed that PTEN was a target gene of miR23a-3p.

GAS5 inhibits osteosarcoma cell proliferation and invasion via miR-23a-3p/PTEN/PI3K/AKT pathway in U2OS and HOS cells

To explore the relationship among GAS5, miR-23a-3p and PTEN, the U2OS and HOS cells were transfected with Vector, pcDNA-GAS5, pcDNA-GAS5 and NC-mimic, pcDNA-GAS5 and miR-23a-3p mimic, and pcDNA-GAS5 and Scramble, or pcDNA-GAS5 and si-PTEN. Cell proliferation results showed that overexpression of GAS5 could inhibit cell growth, while overexpression of miR-23a-3p or silence of PTEN could reversed the inhibitory effect by GAS5 (Fig. 7A and B). Data in Fig.7C-F showed that cells cotransfected with pcDNA-GAS5 and NC-mimic significant decreased cell invasion but increased cell apoptotic rate $(P<0.01)$. However, co-transfection with pcDNA-GAS5 and miR-23a-3p mimic or pcDNAGAS5 and si-PTEN could counteract the decrease of cell invasion and the increase of cell apoptosis. As shown in Fig.7 $\mathrm{G}$ and $\mathrm{H}$, cells co-transfected with pcDNA-GAS5 and NC-mimic or pcDNA-GAS5 and Scramble could signally increase the protein level of PTEN $(P<0.01)$, while reduce the phosphorylated 
levels of PI3K and AKT $(P<0.01)$. However, in relation to the Vector group, the protein levels of PTEN and phosphorylated PI3K and AKT did not change remarkably. These data suggested that overexpression of GAS5 inhibited osteosarcoma cell migration and invasion by downregulating the expression of miR-23a$3 p$ and upregulating PTEN level to inhibit the activation of PI3K/AKT pathway in osteosarcoma cells.

\section{Overexpression of GAS5 inhibits the tumorigenesis of osteosarcoma in vivo}

In vivo experiments, overexpression of GAS5 could significantly suppress tumor growth. Compared with Vector group, pcDNA-GAS5 group had smaller tumor size and lighter weight (Fig. 8A-C). RT-qPCR data demonstrated that the level of GAS5 was higher than that in Vector group, while miR-23a-3p expression was reduced (Fig. 8D and E). Western blot results showed that the protein expression of PTEN was increased in pcDNA-GAS5 group (Fig. 8F). These findings suggest that the antitumor effect of GAS5 was mediated by miR-23a-3p and PTEN in osteosarcoma.

\section{Discussion}

Disorders of IncRNAs have been shown to be involved in the development and progression of many human diseases. GAS5 also plays an important regulatory role in pathophysiological process. For instance, GAS5 was upregulated in atherosclerosis, suggesting that GAS5 implicated in the development of atherosclerosis[25]. Moreover, the expression levels of GAS5 was remarkably increased in epilepsy and ischaemic heart disease[26, 27]. In this study, we found that GAS5 expression significantly downregulated in osteosarcoma, which indicated that GAS5 was involved in osteosarcoma metastasis, and some studies had similar results. In gastric cancer, GAS5 was downregulated in tissues and cell lines[28], and GAS5 also was downregulated in diabetic nephropathy[29]. The inconsistence may be attributed to the different pathogenesis of different diseases.

Overexpression of GAS5 could inhibit the proliferation, invasion and promote apoptosis of osteosarcoma cells, which fully indicated that GSA5 acted as a tumor suppressor in osteosarcoma cells. However, the mechanism how GAS5 inhibited osteosarcoma growth and metabolism was unclear. GAS5 has been reported to interact with miRNAs to participate in the process of tumorigenesis. Reports had indicated that GAS5 inhibited laryngeal squamous cell proliferation by reversing ectopic expression of miR-21[30]. Other reports revealed that GAS5 prevented human B lymphocytic leukaemia tumourigenesis and metastasis by sponging miR-222[31]. However, we found that GAS5 could interact with miR-23a-3p through luciferase reporter gene and RNA immunoprecipitation. It has been demonstrated that miR-23a$3 p$ could act as an oncogene[22]. Overexpression GAS5 could suppress the expression of miR-23a-3p. Therefore, we hypothesized that GAS5 may inhibit tumor proliferation and invasion by inhibiting the expression of miR-23a-3p.

It has been suggested that miRNAs could post-transcriptionally control genes expression by targeting the 3'-untranslated regions (UTR) mRNAs. Studies have reported that the expression and function of miR-23a$3 p$ are different in various pathological tissues. MiR-23a-3p was downregulated in oral squamous cell carcinomas and inhibited the expression of fibroblast growth factor 2 (FGF2) to suppress cell 
proliferation [32]. However, high expression of miR-23a-3p promoted tumorigenesis by inhibiting ProlineRich Nuclear Receptor Coactivator 2 (PNRC2) in renal cell carcinoma[22]. PTEN is well known to function as a tumor suppressor gene in the initiation and development of cancer. In this study, we conducted luciferase reporter gene assay to validate that PTEN was a target gene of miR-23a-3p. And miR-23a-3p could target and downregulate the level of PTEN in osteosarcoma cells.

Signaling pathway plays an important role in the occurrence and development of human tumors. Previous studies had displayed that GAS5 might inhibit the proliferation of mesangial cells and accumulation of extracellular matrix via nuclear transcription factor-kB (NF-kB) pathway[33]. Song et al reported that GAS5 inhibited angiogenesis and metastasis of colorectal cancer through the Wnt/ $\beta$ catenin signaling pathway[34]. PTEN mainly acts on the downstream target molecule PIP3 of PI3K through its lipid phosphatase activity, thereby blocking the PI3K/AKT signaling pathway to achieve its anticancer effect. Therefore, in this paper, we wondered whether GAS5 could inhibit cell proliferation and invasion in osteosarcoma cells through the PTEN/PI3K/AKT signaling pathway. The findings revealed that overexpression of GAS5 suppressed the levels of miR-23a-3p, p-PI3K and p-AKT, while increased the level of PTEN, suggesting that GAS5 was involved in the development of osteosarcoma through $\mathrm{PI3K} / \mathrm{AKT}$ signaling pathway.

In vivo xenograft experiments showed that overexpression GAS5 could suppress the growth of osteosarcoma and the expression of miR-23a-3p, while upregulate the level of PTEN.

\section{Conclusions}

Based on the findings acquired from this study, overexpression GAS5 could inhibit osteosarcoma cell proliferation and invasion in vitro and suppress tumor growth in vivo through the miR-23a3p/PTEN/PI3K/AKT axis. Targeting the GAS5/miR-23a-3p/PTEN axis may represent a novel therapeutic perspective, contributing to a better knowledge of the molecular mechanism of osteosarcoma.

\section{Abbreviations}

growth arrest special 5 (GAS5); phosphatase and tensin homolog (PTEN); long noncoding RNAs (IncRNAs) ; competing endogenous RNAs (ceRNAs); Reverse Transcription-quantitative PCR (RT-qPCR) ; Argonaute2 (Ago2); RNA immunoprecipitation (RIP); sodium dodecyl sulfate polyacrylamide gel electrophoresis (SDS-PAGE); polyvinylidene difluoride (PVDF) membranes; phosphatidylinositol-3kinases/protein-serine-threonine kinase (PI3K/AKT); nuclear transcription factor-KB (NF-KB); Proline-Rich Nuclear Receptor Coactivator 2 (PNRC2); fibroblast growth factor 2 (FGF2); untranslated regions (UTR).

\section{Declarations}

\section{Ethics approval and consent to participate}


All patients had not received preoperative radiotherapy and/or chemotherapy and were obtained with informed consent before surgery. The protocols were approved by the Ethics Committee of Shaanxi Provincial People's Hospital.

\section{Consent for publication}

All the author agreed to this submission and approved the publication.

\section{Competing interests}

The authors have declared that they have no competing interests.

\section{Funding}

No funding was received.

\section{Availability of date materials}

The datasets used during the present study are available from the corresponding author upon reasonable request.

\section{Acknowledgements}

Not applicable.

\section{Authors' contributions}

Liu performed the experiments and prepared the manuscript; M. Chen designed the study; L. Ma and Xingbo Dang prepared the figures; $G$. Du performed the statistical analysis.

\section{References}

1. Wei CJ, Li YL, Zhu ZL, Jia DM, Fan ML, Li T, Wang XJ, Li ZG, Ma HS: Inhibition of activator protein 1 attenuates neuroinflammation and brain injury after experimental intracerebral hemorrhage. $C N S$ Neurosci Ther2019, 25(10):1182-1188.

2. Fei D, Zhang X, Liu J, Tan L, Xing J, Zhao D, Zhang Y: Long Noncoding RNA FER1L4 Suppresses Tumorigenesis by Regulating the Expression of PTEN Targeting miR-18a-5p in Osteosarcoma. (14219778 (Electronic)).

3. Li WT, Zhang Q: MicroRNA-708-5p regulates mycobacterial vitality and the secretion of inflammatory factors in Mycobacterium tuberculosis-infected macrophages by targeting TLR4. Eur Rev Med Pharmacol Sci 2019, 23(18):8028-8038. 
4. Gu Q, Luo Y, Chen C, Jiang D, Huang Q, Wang X: GREM1 overexpression inhibits proliferation, migration and angiogenesis of osteosarcoma. (1090-2422 (Electronic)).

5. Wang Y Fau - Kong D, Kong D: LncRNA GAS5 Represses Osteosarcoma Cells Growth and Metastasis via Sponging MiR-203a. (1421-9778 (Electronic)).

6. Wang W, Li J, Ding Z, Li Y, Wang J, Chen S, Miao JA-Ohoo: Tanshinone I inhibits the growth and metastasis of osteosarcoma via suppressing JAK/STAT3 signalling pathway. (1582-4934 (Electronic)).

7. Zhang X, Du K, Lou Z, Ding K, Zhang F, Zhu J, Chang Z: The CtBP1-HDAC1/2-IRF1 transcriptional complex represses the expression of the long noncoding RNA GAS5 in human osteosarcoma cells. (1449-2288 (Electronic)).

8. Xin Y, Lyu X, Wang C, Fu Y, Zhang S, Tian C, Li Q, Zhang D: Elevated circulating levels of CTRP1, a novel adipokine, in diabetic patients. Endocr J 2014, 61(9):841-847.

9. Lu L, Zhang RY, Wang XQ, Liu ZH, Shen Y, Ding FH, Meng H, Wang LJ, Yan XX, Yang K et al: C1q/TNFrelated protein-1: an adipokine marking and promoting atherosclerosis. Eur Heart J 2016, 37(22):1762-1771.

10. Wen Q, Liu Y Fau - Lyu H, Lyu H Fau - Xu X, Xu X Fau - Wu Q, Wu Q Fau - Liu N, Liu N Fau - Yin Q, Yin Q Fau - Li J, Li J Fau - Sheng X, Sheng X: Long Noncoding RNA GAS5, Which Acts as a Tumor Suppressor via microRNA 21, Regulates Cisplatin Resistance Expression in Cervical Cancer. (15251438 (Electronic)).

11. Gao ZQ, Wang JF, Chen DH, Ma XS, Wu Y, Tang Z, Dang XW: Long non-coding RNA GAS5 suppresses pancreatic cancer metastasis through modulating miR-32-5p/PTEN axis. (2045-3701 (Print)).

12. Dong S, Zhang XA-Ohoo, Liu D: Overexpression of long noncoding RNA GAS5 suppresses tumorigenesis and development of gastric cancer by sponging miR-106a-5p through the Akt/mTOR pathway. LID - bio041343 [pii] LID - 10.1242/bio.041343 [doi]. (2046-6390 (Print)).

13. Wang Y, Kong D: LncRNA GAS5 Represses Osteosarcoma Cells Growth and Metastasis via Sponging MiR-203a. Cellular Physiology \& Biochemistry:844-855.

14. Fan YZ, Huang H, Wang S, Tan GJ, Zhang QZ: Effect of IncRNA MALAT1 on rats with myocardial infarction through regulating ERK/MAPK signaling pathway. Eur Rev Med Pharmacol Sci 2019, 23(20):9041-9049.

15. Vo DT, Karanam NK, Ding L, Saha D, Yordy JS, Giri U, Heymach JV, Story MD: miR-125a-5p Functions as Tumor Suppressor microRNA And Is a Marker of Locoregional Recurrence And Poor prognosis in Head And Neck Cancer. (1476-5586 (Electronic)).

16. Zhang Y, Zhang D, Lv J, Wang S, Zhang Q: MiR-125a-5p suppresses bladder cancer progression through targeting FUT4. (1950-6007 (Electronic)).

17. Jia J, Zhang M, Li Q, Zhou Q, Jiang Y: Long noncoding ribonucleic acid NKILA induces the endoplasmic reticulum stress/autophagy pathway and inhibits the nuclear factor-k-gene binding pathway in rats after intracerebral hemorrhage. J Cell Physio/ 2018, 233(11):8839-8849. 
18. Sun L, Liu M, Luan S, Shi Y, Wang Q: MicroRNA-744 promotes carcinogenesis in osteosarcoma through targeting LATS2. (1792-1074 (Print)).

19. Dorn GW, 2nd, Matkovich SJ: Menage a Trois: intimate relationship among a microRNA, long noncoding RNA, and mRNA. (1524-4571 (Electronic)).

20. Cui HA-Ohoo, Zhao J: LncRNA TMPO-AS1 serves as a ceRNA to promote osteosarcoma tumorigenesis by regulating miR-199a-5p/WNT7B axis. LID - 10.1002/jcb.29451 [doi]. (1097-4644 (Electronic)).

21. Li K, Zhao B, Wei D, Cui Y, Qian L, Wang WA-Ohoo, Liu G: Long non-coding RNA ANRIL enhances mitochondrial function of hepatocellular carcinoma by regulating the MiR-199a-5p/ARL2 axis. LID 10.1002/tox.22867 [doi]. (1522-7278 (Electronic)).

22. Quan J, Pan X, Li Y, Hu Y, Tao L, Li Z, Zhao L, Wang J, Li H, Lai Y et al: MiR-23a-3p acts as an oncogene and potential prognostic biomarker by targeting PNRC2 in RCC. (1950-6007 (Electronic)).

23. Chen G, Li Y, He Y, Zeng B, Yi C, Wang C, Zhang X, Zhao W, Yu D: Upregulation of Circular RNA circATRNL1 to Sensitize Oral Squamous Cell Carcinoma to Irradiation. Mol Ther Nucleic Acids 2020, 19:961-973.

24. Jalali S, Bhartiya D Fau - Lalwani MK, Lalwani Mk Fau - Sivasubbu S, Sivasubbu S Fau - Scaria V, Scaria V: Systematic transcriptome wide analysis of IncRNA-miRNA interactions. (1932-6203 (Electronic)).

25. Lewis Ta Fau - Taylor FR, Taylor Fr Fau - Parks LW, Parks LW: Involvement of heme biosynthesis in control of sterol uptake by Saccharomyces cerevisiae. (0021-9193 (Print)).

26. Li BG, Wu WJA-Ohoo, Zheng HC, Yang HF, Zuo YX, Cui XP: Long noncoding RNA GAS5 silencing inhibits the expression of KCNQ3 by sponging miR-135a-5p to prevent the progression of epilepsy. (2410-8650 (Electronic)).

27. Wu NA-Ohoo, Zhang X, Bao Y, Yu H, Jia D, Ma C: Down-regulation of GAS5 ameliorates myocardial ischaemia/reperfusion injury via the miR-335/ROCK1/AKT/GSK-3beta axis. LID 10.1111/jcmm.14724 [doi]. (1582-4934 (Electronic)).

28. Liu Y, Yin L, Chen C, Zhang X, Wang S: Long non-coding RNA GAS5 inhibits migration and invasion in gastric cancer via interacting with p53 protein. LID - S1590-8658(19)30735-2 [pii] LID 10.1016/j.dld.2019.08.012 [doi]. (1878-3562 (Electronic)).

29. Ge X, Xu B, Xu W, Xia L, Xu Z, Shen L, Peng W, Huang S: Long noncoding RNA GAS5 inhibits cell proliferation and fibrosis in diabetic nephropathy by sponging miR-221 and modulating SIRT1 expression. (1945-4589 (Electronic)).

30. Lyu K, Xu Y, Yue H, Li Y, Zhao J, Chen L, Wu J, Zhu X, Chai L, Li C et al: Long Noncoding RNA GAS5 Acts As A Tumor Suppressor In Laryngeal Squamous Cell Carcinoma Via miR-21. (1179-1322 (Print)).

31. Jing Z, Gao L, Wang H, Chen J, Nie B, Hong Q: Long non-coding RNA GAS5 regulates human B lymphocytic leukaemia tumourigenesis and metastasis by sponging miR-222. LID - 10.3233/cbm190246 [doi]. (1875-8592 (Electronic)). 
32. Chen F, Qi S, Zhang X, Wu J, Yang X, Wang R: miR-23a-3p suppresses cell proliferation in oral squamous cell carcinomas by targeting FGF2 and correlates with a better prognosis: miR-23a-3p inhibits OSCC growth by targeting FGF2. (1618-0631 (Electronic)).

33. Zhang $R$, Han $X$, Huang $T$, Wang $X$ : Danggui buxue tang inhibited mesangial cell proliferation and extracellular matrix accumulation through GAS5/NF-kappaB pathway. LID - BSR20181740 [pii] LID 10.1042/BSR20181740 [doi]. (1573-4935 (Electronic)).

34. Song J, Shu H, Zhang L, Xiong JA-Ohoo: Long noncoding RNA GAS5 inhibits angiogenesis and metastasis of colorectal cancer through the Wnt/beta-catenin signaling pathway. LID 10.1002/jcb.27743 [doi]. (1097-4644 (Electronic)).

\section{Figures}
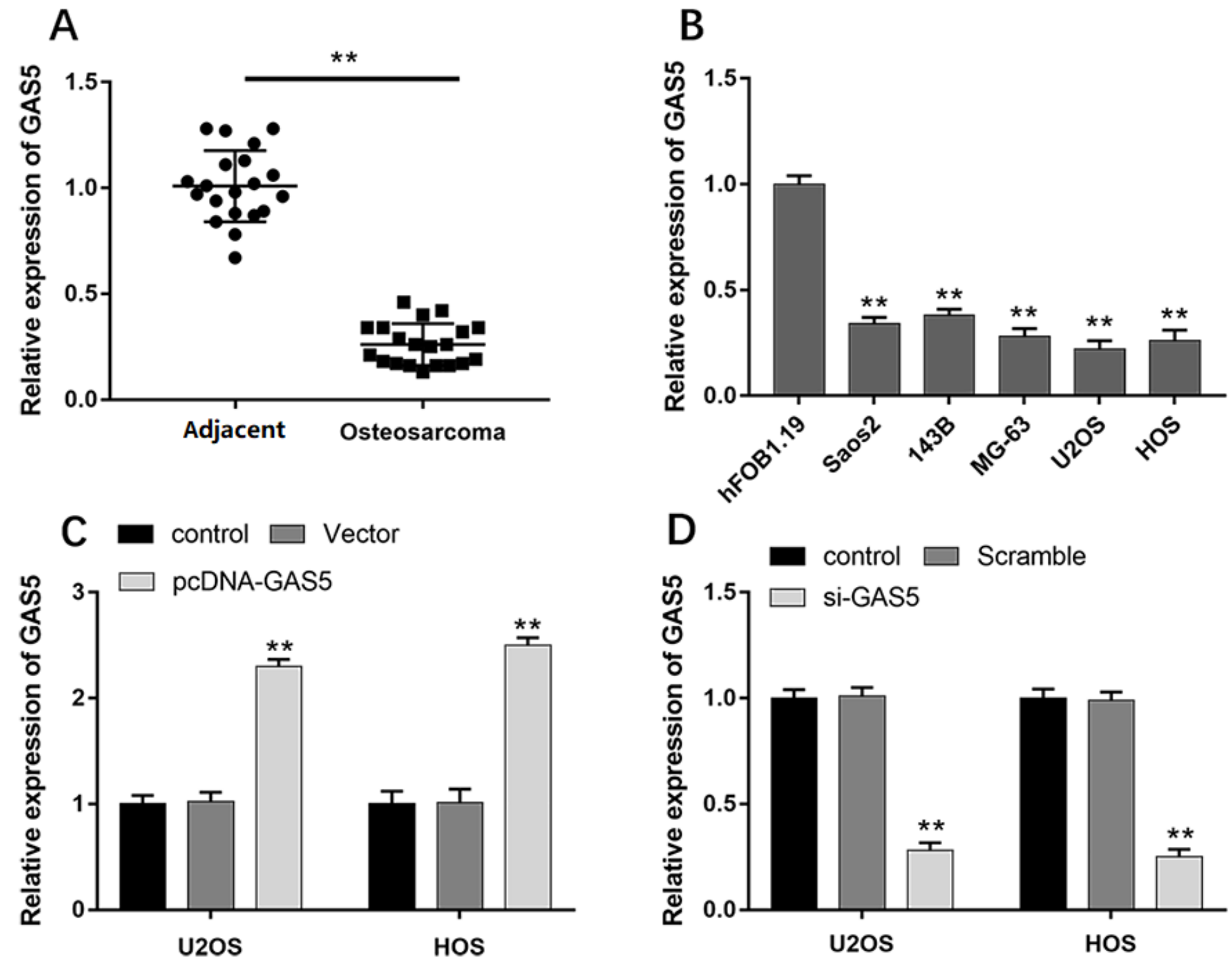

Figure 1 
LncRNA GAS5 is downregulated in osteosarcoma tissues and cell lines. (A) The expression of IncRNA GAS5 in human osteosarcoma tissues and normal adjacent tissues were detected by using qPCR $(n=20)$. (B)The levels of GAS5 in Human normal osteoblast cells (hFOB1.19) and human OS cell lines (Saos2, 143B, MG-63, U2OS, HOS) were measured by using qPCR. (C) The level of GAS5 was detected after transfection with pcDNA-GAS5 for $24 \mathrm{~h}$. (D) The expression of GAS5 was detected after transfection with si-GAS5 for 24 h. ** $\mathrm{P}<0.01$ compared with adjacent tissues or hFOB1.19 cells.

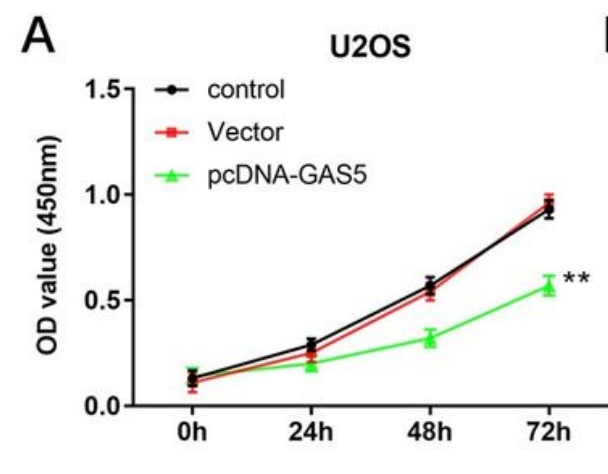

D
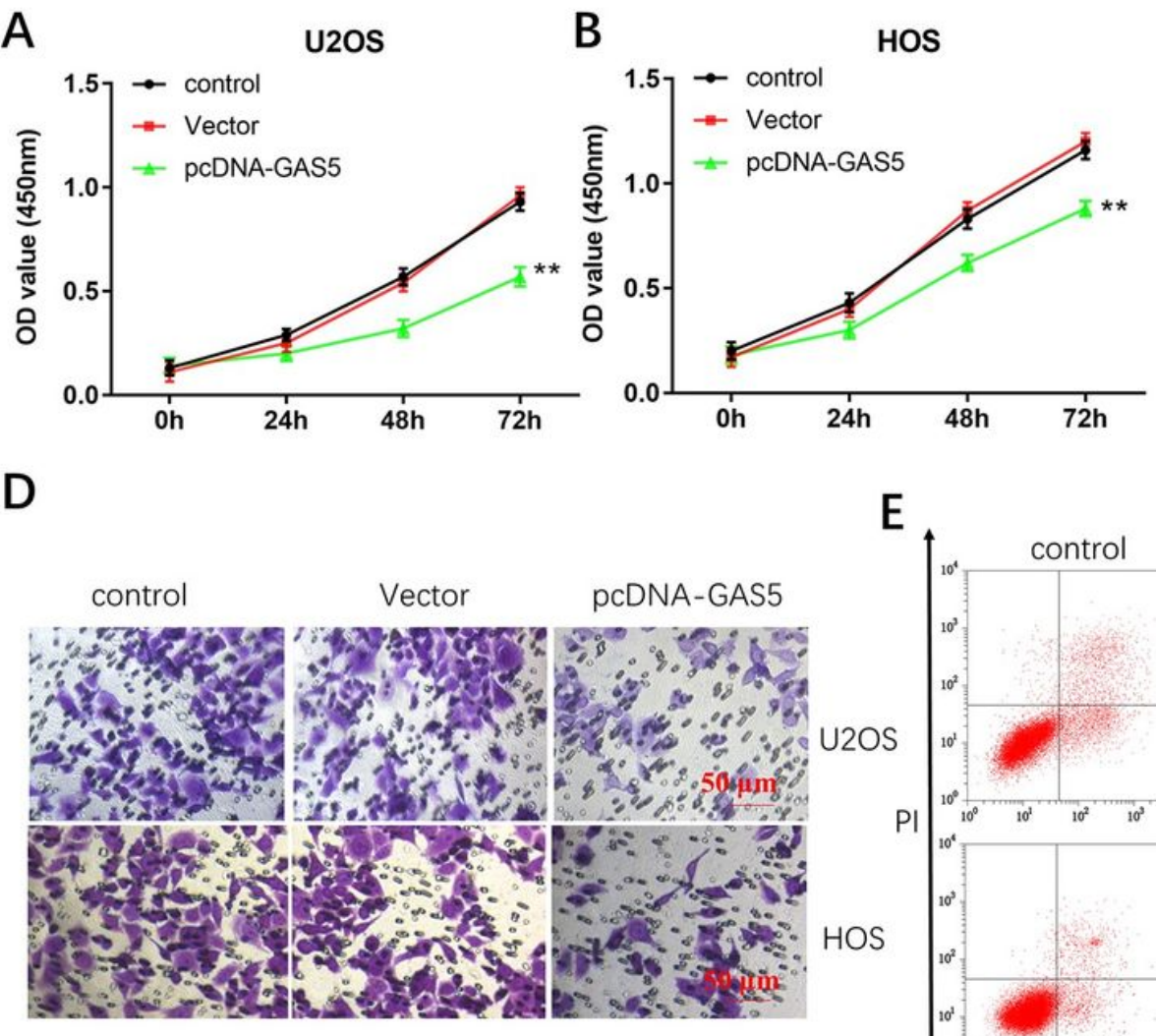

C $\quad$ pcontrol $\square$ Vector

E
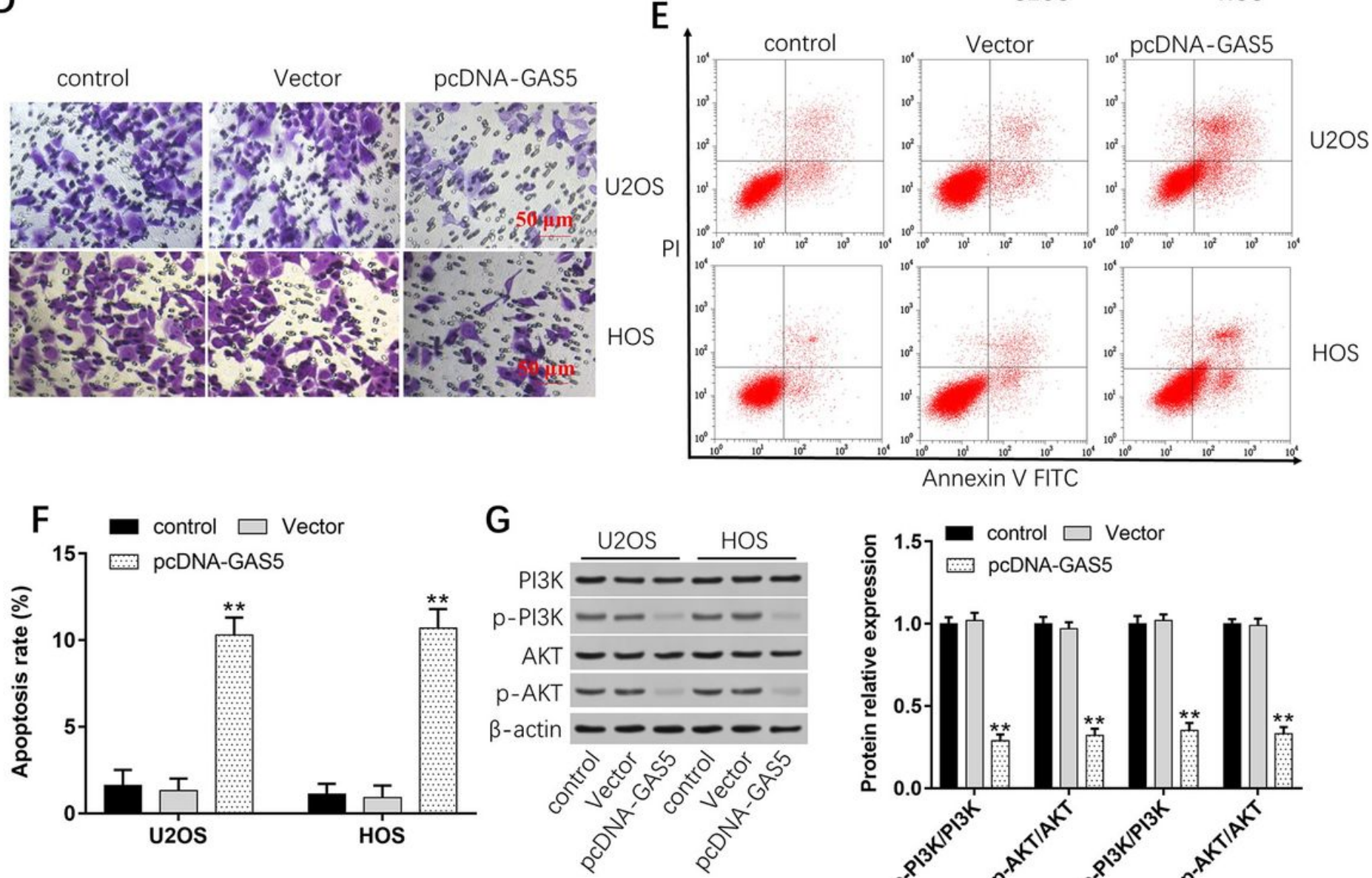
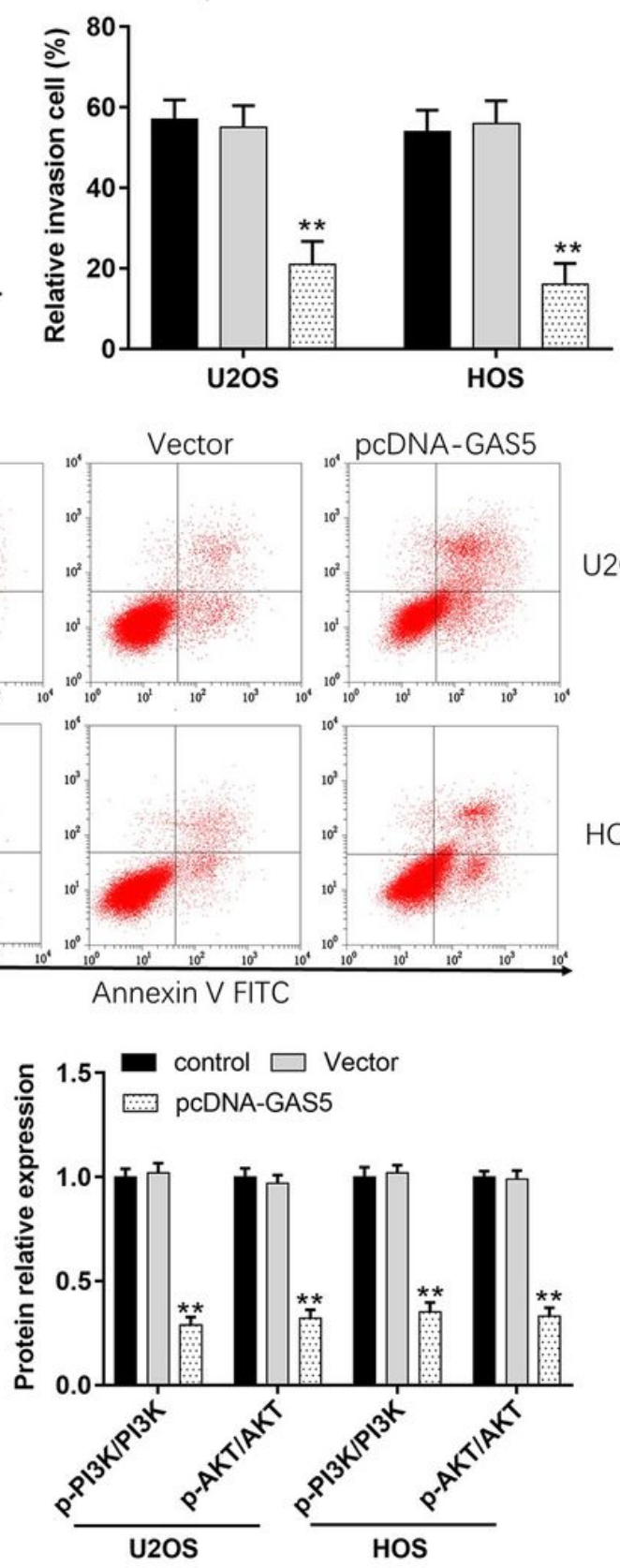

Figure 2

Overexpression GAS5 inhibits cell growth, invasion and PI3K/AKT signal pathway. (A-B) Cell proliferation of U2OS and HOS cells were detected by using CCK-8 assay after transfection with pcDNA-GAS5 for 0,24 , 
48 and 72h. (C-D) The invasion ability of U2OS and HOS cells were evaluated by using Transwell invasion assay after transfection with pcDNA-GAS5 for $48 \mathrm{~h}$. (E-F) Apoptosis rates of U2OS and HOS cells after transfection with pcDNA-GAS5 were detected by using Flow cytometry. (G) Western blot analysis was used to detect the protein expression levels of PI3K, p-PI3K, AKT, p-AKT after transfection with pcDNAGAS5. *P $<0.05$ and $* * P<0.01$ compared with control.

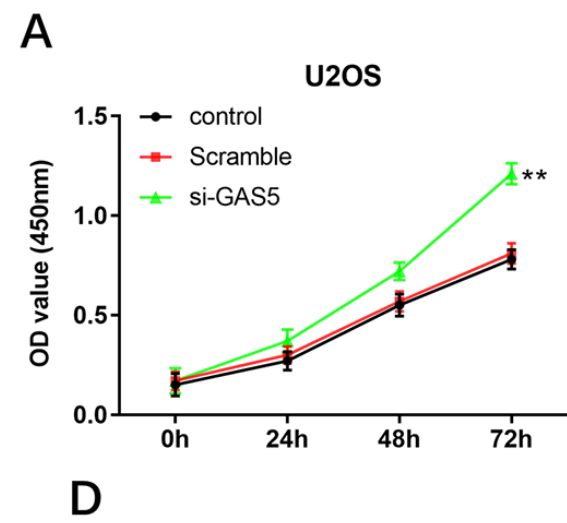

B

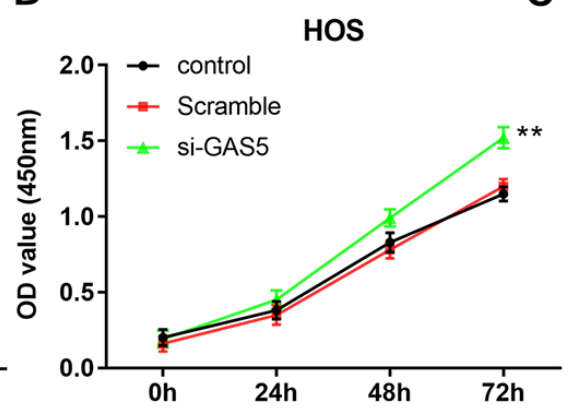

$\mathrm{E}$
C

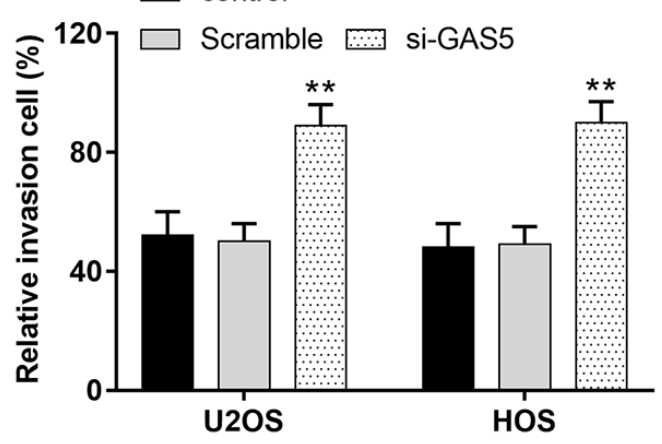

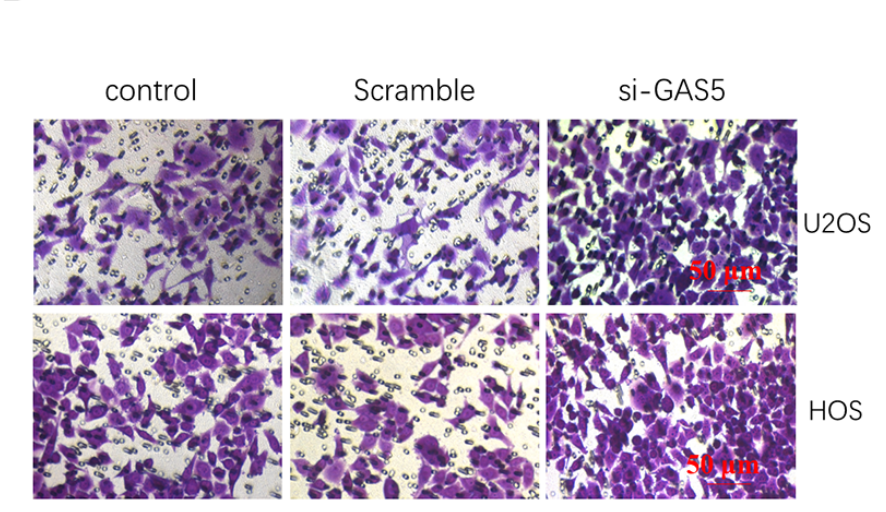

$\hat{10}^{10}$ control Scramble
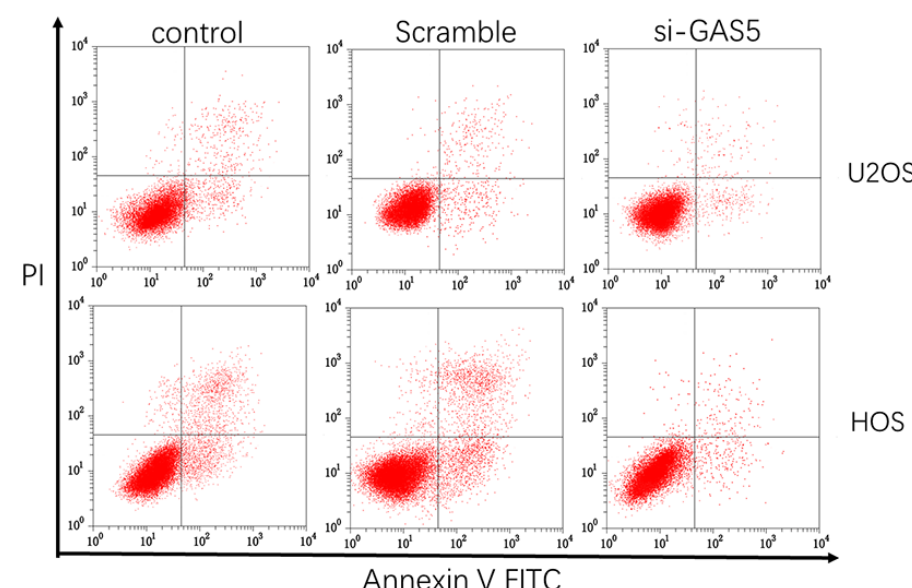

$\mathrm{U} 2 \mathrm{OS}$

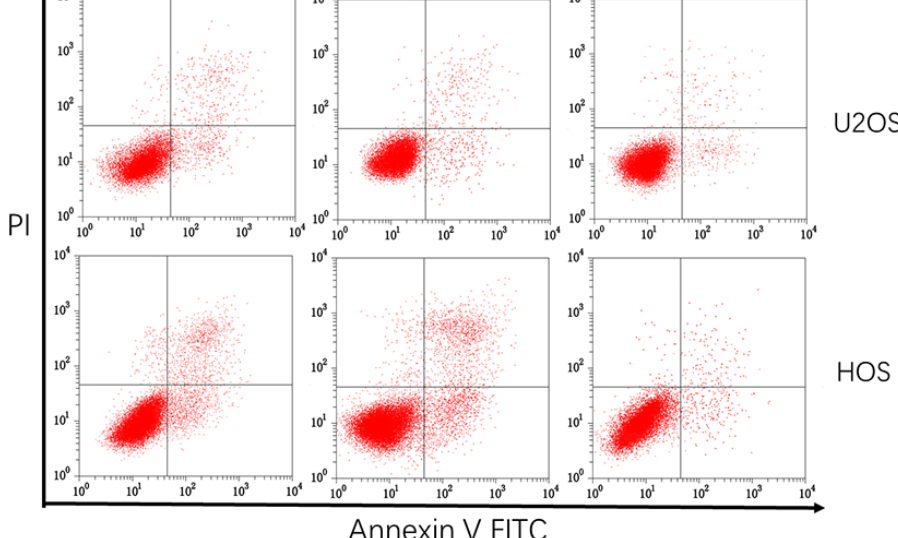

Annexin V FITC

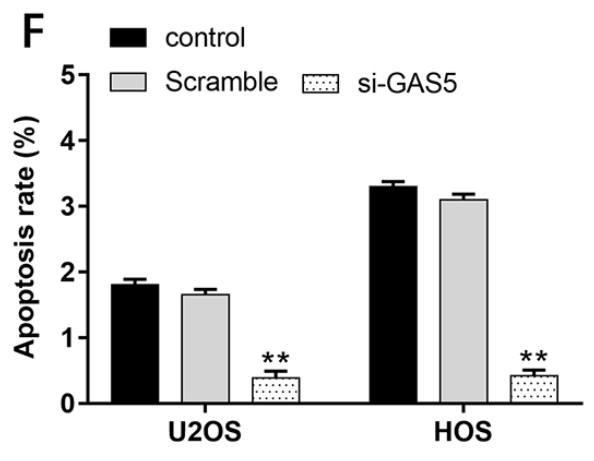

G
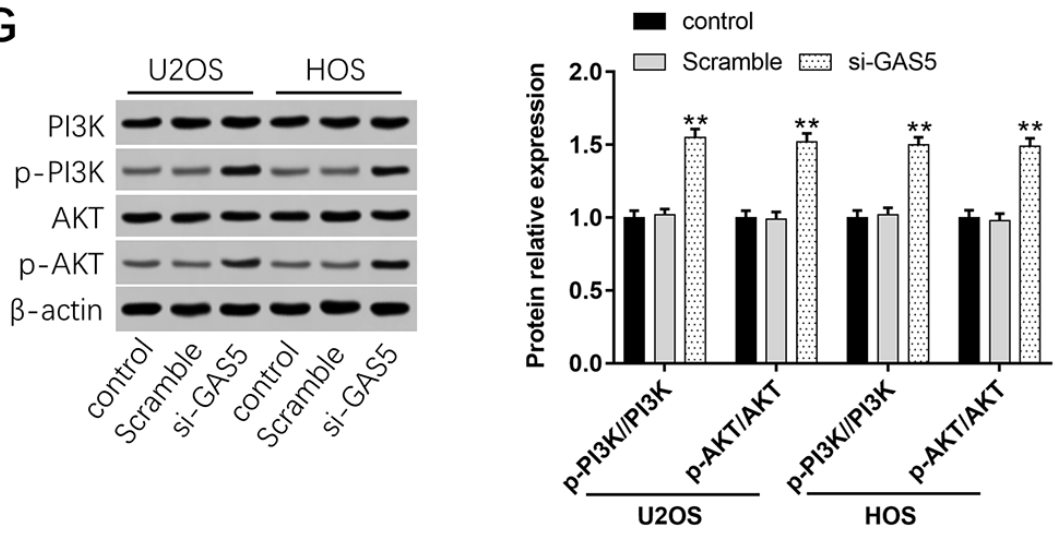

\section{Figure 3}

Downregulation GAS5 promotes cell growth, invasion and activates PI3K/AKT signal pathway. (A-B) Cell proliferation of U2OS and HOS cells were detected by using CCK-8 assay after transfection with si-GAS5 for $0,24,48$ and $72 \mathrm{~h}$. (C-D) The invasion ability of U2OS and HOS cells were evaluated by using Transwell invasion assay after transfection with si-GAS5 for $48 \mathrm{~h}$. (E-F) Apoptosis rates of U2OS and HOS cells after transfection with si-GAS5 were detected by using Flow cytometry. (G) Western blot analysis 
was used to detect the protein expression levels of PI3K, p-PI3K, AKT, p-AKT after transfection with siGAS5. ** $\mathrm{P}<0.01$ compared with control.

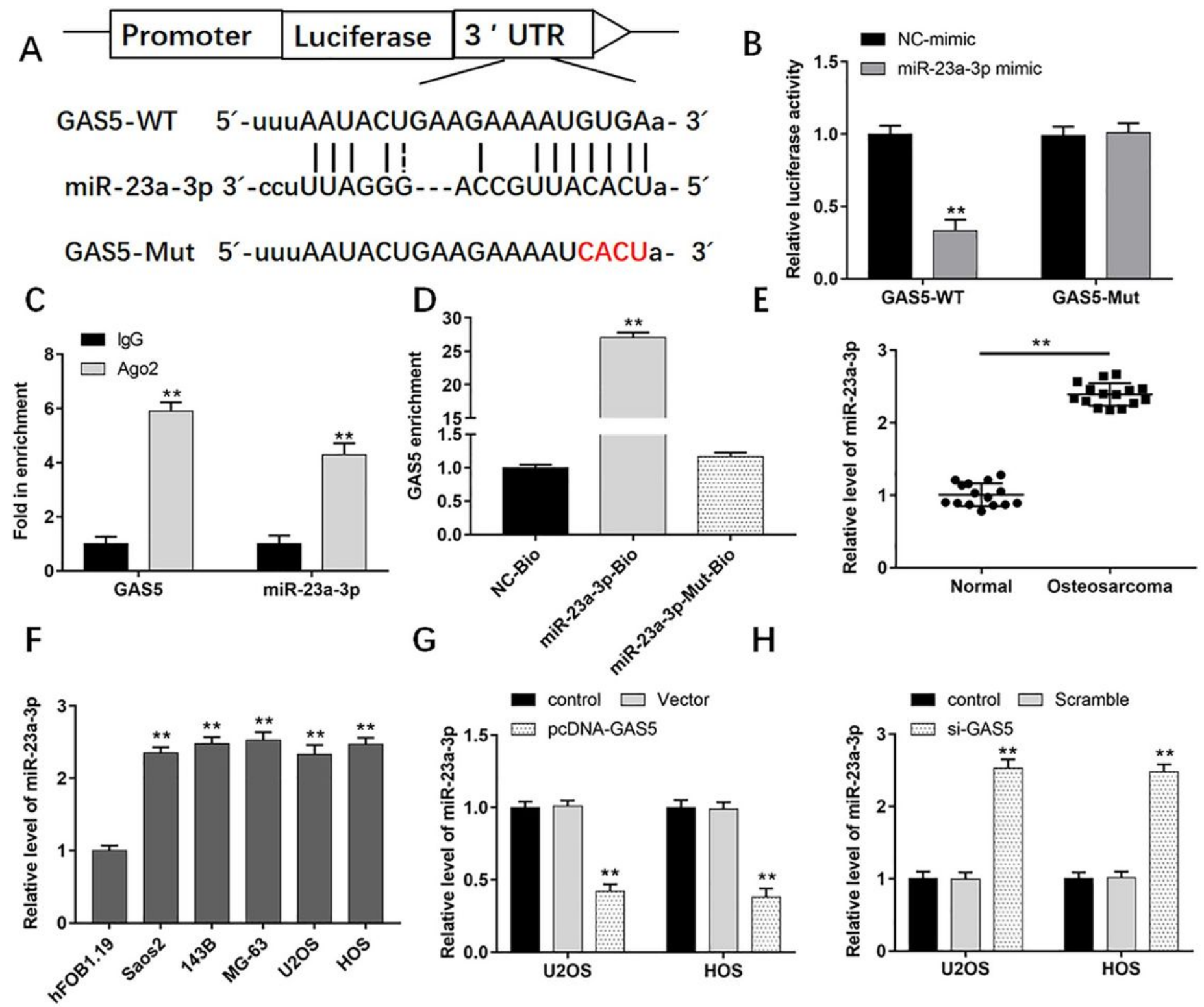

Figure 4

GAS5 is a direct target gene of miR-23a-3p and regulates expression of miR-23a-3p. (A) The binding sites of miR-23a-3p and GAS5. (B) Luciferase activity of GAS5-Wt and GAS5-Mut without or with transfection of miR-23a-3p. (C) RIP assay was conducted to examine miR-23a-3p endogenously associated with GAS5. (D) Fold enrichment of miR-23a-3p expression after RNA pull-down experiment with HEK293t cells extracts in different groups. (E) The expression of miR-23a-3p in human osteosarcoma tissues and normal adjacent tissues were detected by using qPCR $(n=20)$. ( $F)$ The levels of miR-23a-3p in Human normal osteoblast cells (hFOB1.19) and human OS cell lines (Saos2, 143B, MG-63, U2OS, HOS) were measured by using qPCR. (G) The levels of miR-23a-3p in U2OS and HOS cells after transfection with pcDNA-GAS5 were detected by using qPCR. $(\mathrm{H})$ The expression of miR-23a-3p in U2OS and HOS cells 
after transfection with si-GAS5 were detected by using qPCR. ${ }^{*} \mathrm{P}<0.01$ compared with adjacent tissues, hFOB1.19 cells or control.
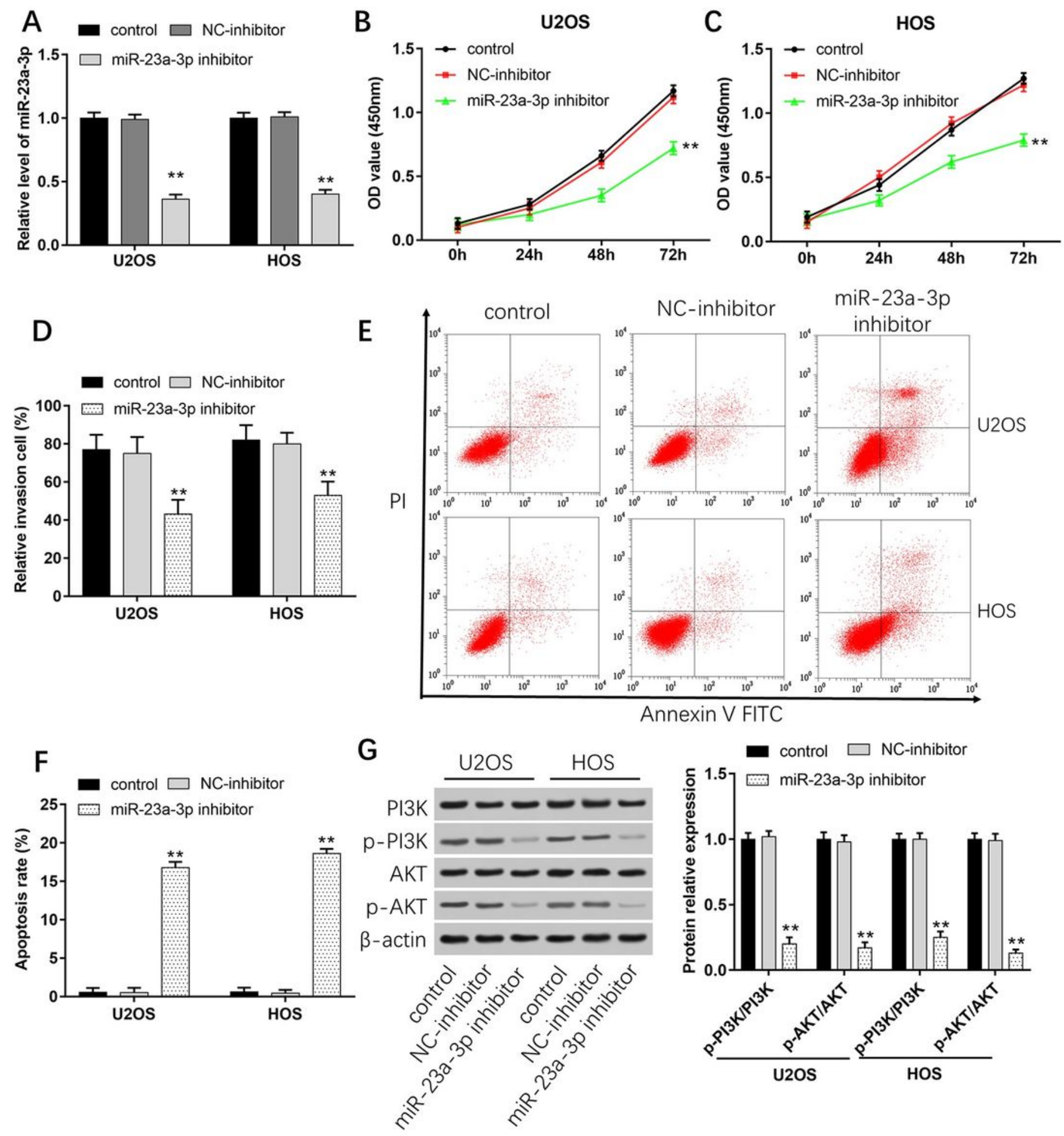

Figure 5

Downregulation of miR-23a-3p inhibits cell growth and invasion through activating PI3K/AKT signal pathway. U2OS and HOS cells were transfected with NC-inhibitor or miR-23a-3p inhibitor. (A) Transfection efficiency was detected by using qPCR. (B-C) Cell proliferation of U2OS and HOS cells were detected by 
using CCK-8 assay. (D) The invasion ability of U2OS and HOS cells were evaluated by using Transwell invasion assay. (E-F) Apoptosis rates of U2OS and HOS cells were detected by using Flow cytometry. (G) Western blot analysis was used to detect the protein expression levels of PI3K, $-\mathrm{PI} 3 \mathrm{~K}, \mathrm{AKT}, \mathrm{p}-\mathrm{AKT}$. ** $\mathrm{P}<$ 0.01 compared with Vector and NC-mimic.
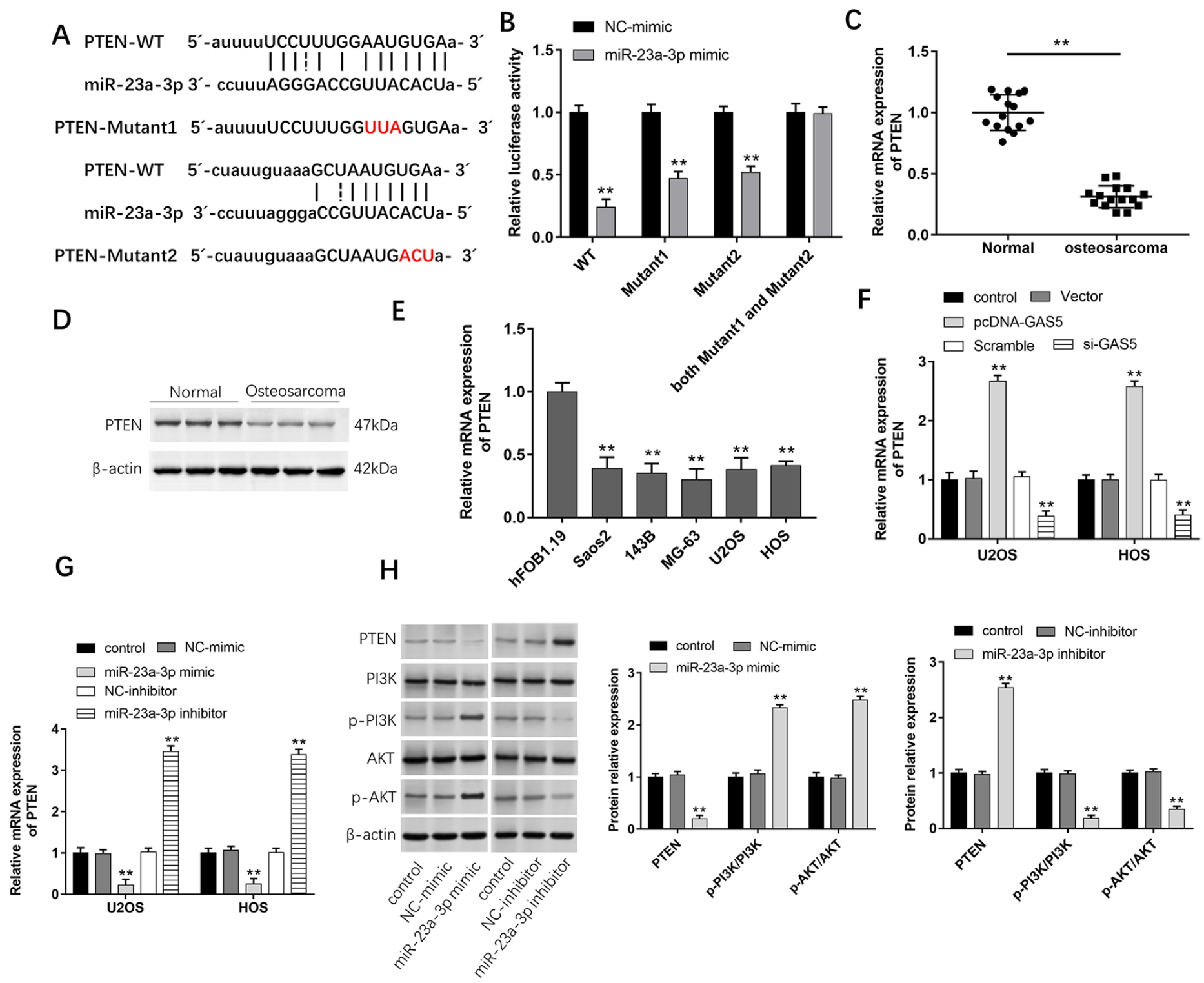

\section{Figure 6}

PTEN is a target gene of miR-23a-3p in osteosarcoma cells. (A) The predicted binding sites between miR23a-3p and PTEN. (B) The relative luciferase activity in HEK-293T cells co-transfected with luciferase reporter vectors containing PTEN -WT, PTEN-Mutant1, PTEN-Mutant2, NC-mimic or miR-23a-3p mimic. (CD) The expression of PTEN in human osteosarcoma tissues and normal adjacent tissues were detected by using qPCR $(n=15)$. (E) The mRNA level of PTEN in Human normal osteoblast cells (hFOB1.19) and human OS cell lines (Saos2, 143B, MG-63, U2OS, HOS). (F-G) The mRNA level of PTEN in U2OS and HOS cells after transfected with pcDNA-GAS5, si-GAS5, miR-23a-3p mimic or miR-23a-3p inhibitor were detected by using qPCR.(H) The protein levels of PTEN, PI3K, p-PI3K, AKT and p-AKT in U2OS cells were 
detected using western blot. ** $\mathrm{P}<0.01$ compared with NC-mimic, normal tissues, hFOB1.19 cells or Vector and NC-mimic.

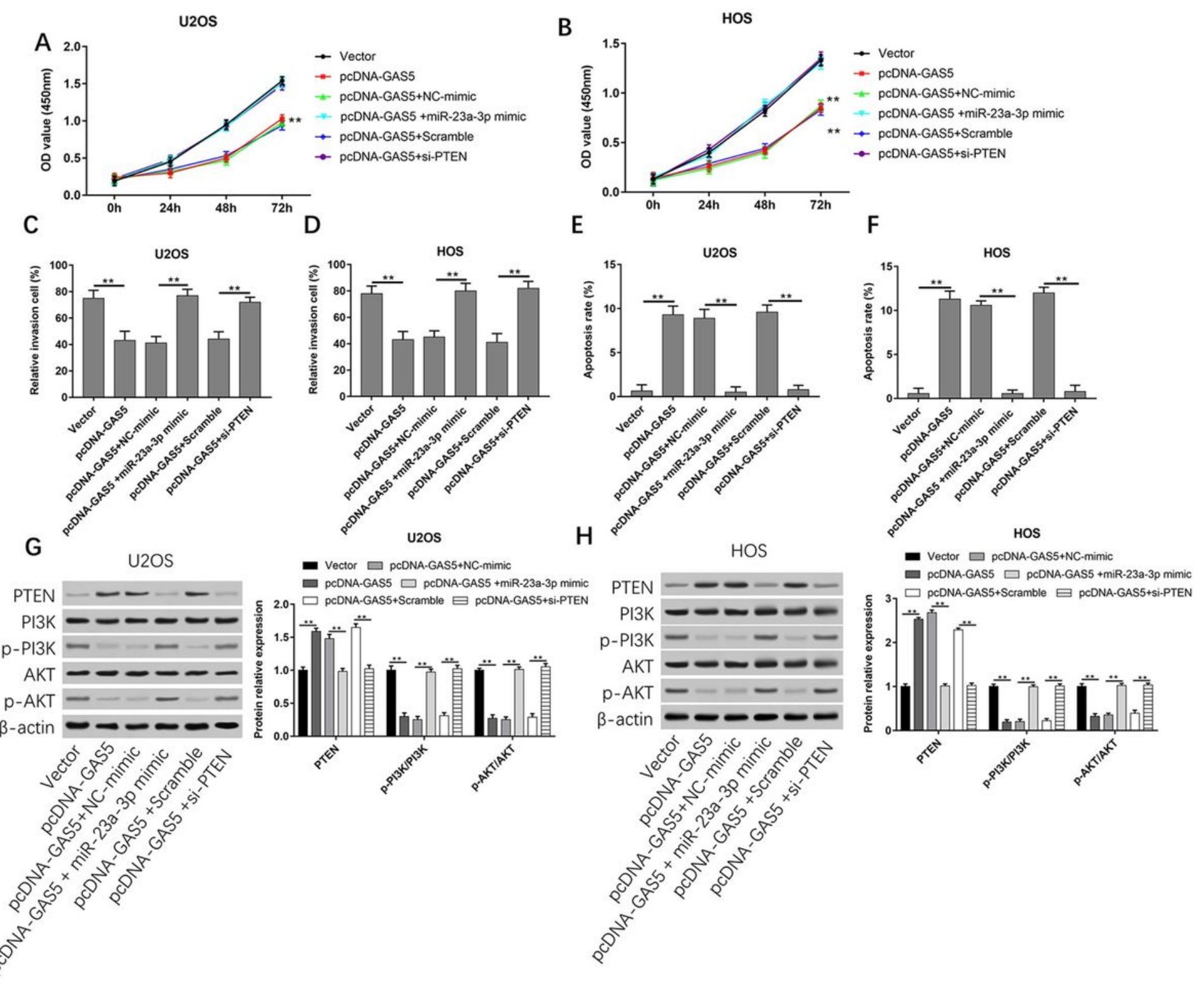

Figure 7

GAS5 inhibits osteosarcoma cell migration and invasion via miR-23a-3p/PTEN/PI3K/AKT axis in U2OS and HOS cells. U2OS and HOS cells were co-transfected with Vector, pcDNA-GAS5, pcDNA-GAS5 and NCmimic, pcDNA-GAS5 and miR-23a-3p mimic, and pcDNA-GAS5 and Scramble, or pcDNA-GAS5 and siPTEN. (A-B) Cell proliferation of U2OS and HOS cells were detected by using CCK-8 assay. (C-D) The invasion ability of U2OS and HOS cells were evaluated by using Transwell invasion assay. (E-F) Apoptosis rates of U2OS and HOS cells were detected by using Flow cytometry. (G-H) Western blot analysis was used to detect the protein expression levels of PTEN, PI3K, p-PI3K, AKT, p-AKT. ** P $<0.01$ compared with Vector and Scramble. 


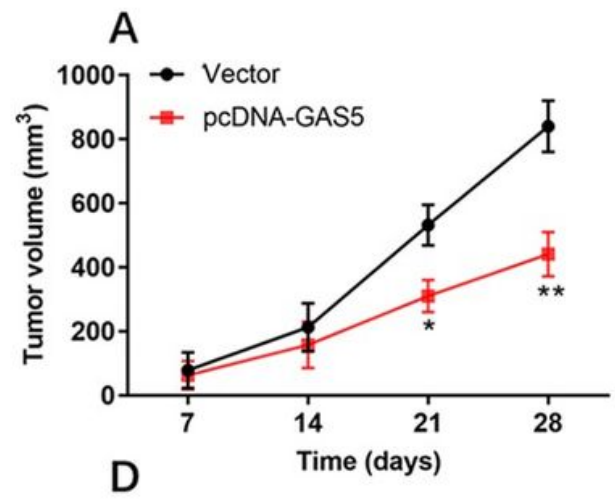

B
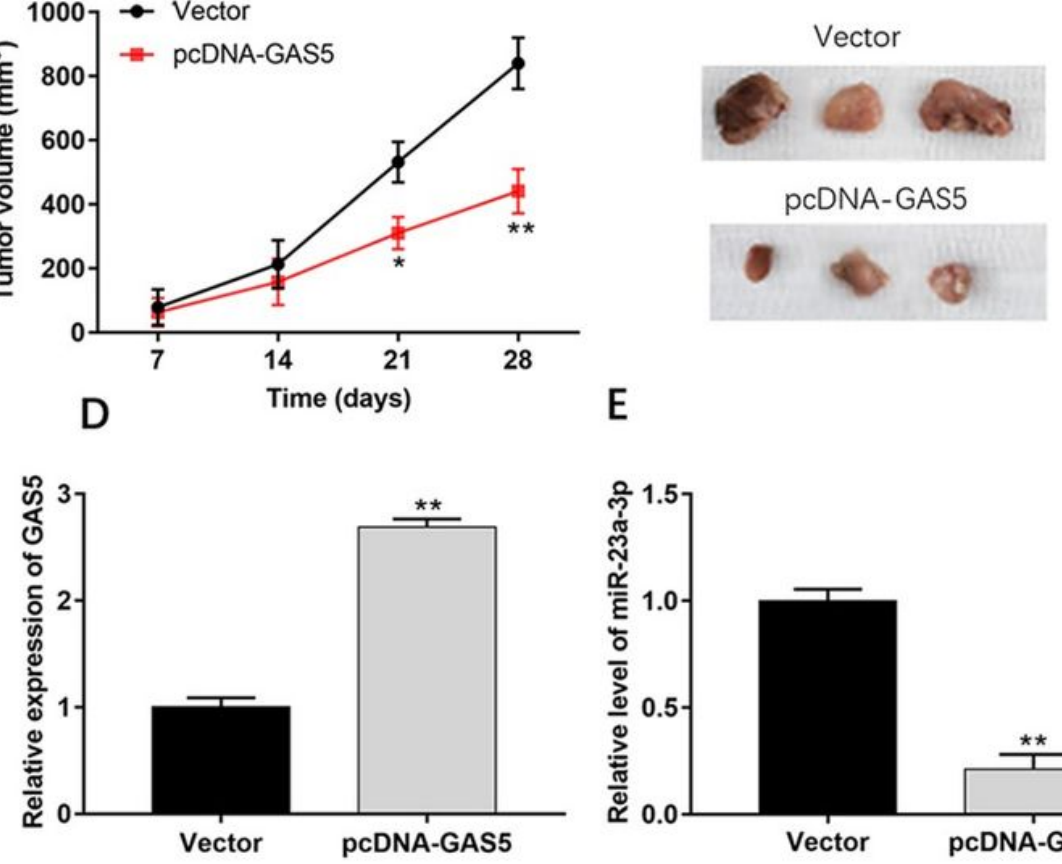

C
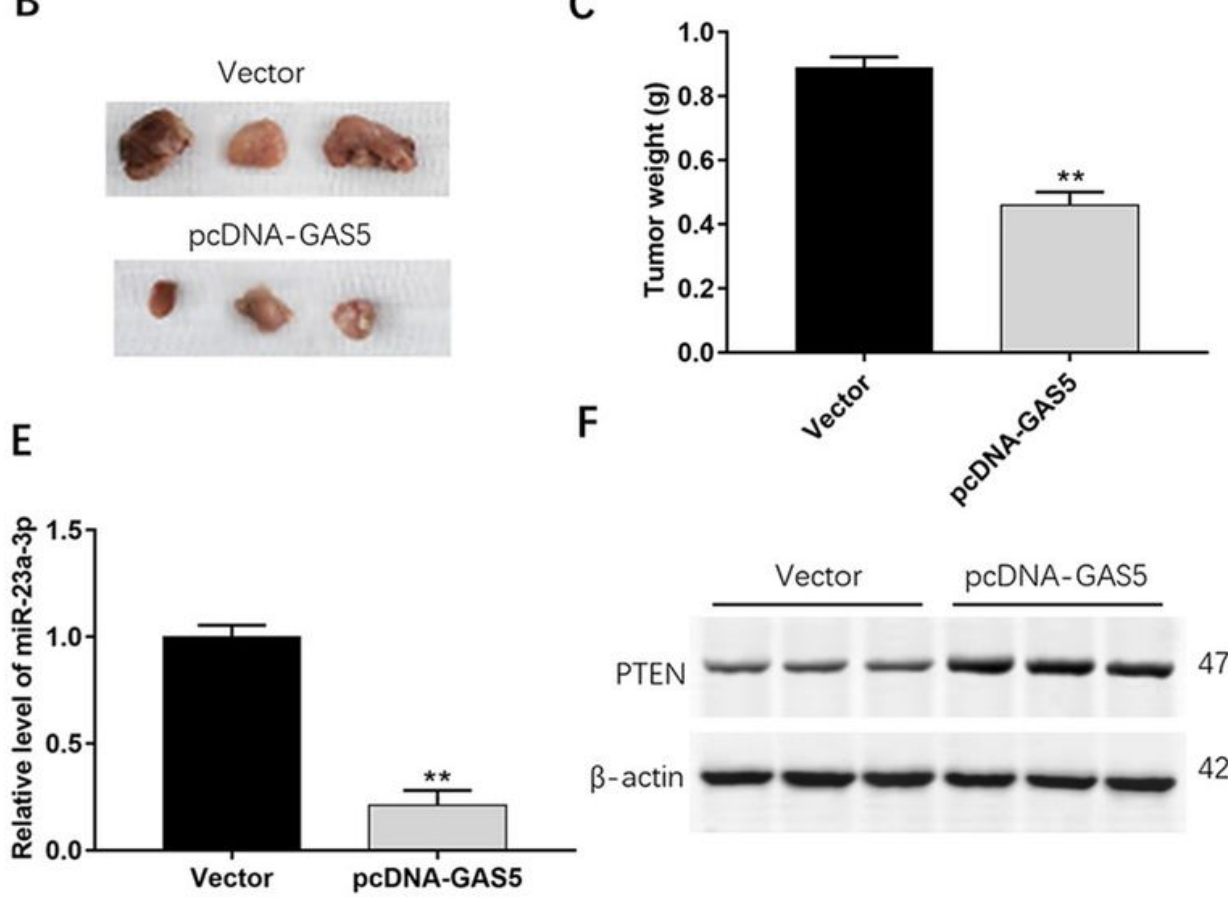

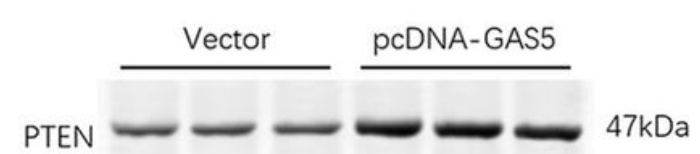

$\beta$-actin

Figure 8

Overexpression of GAS5 inhibits the tumorigenesis of osteosarcoma in vivo. Xenograft tumor model Subcutaneous tumor transplantation model was established by injection with U2OS cells that were permanently transfected with empty vector or pcDNA-GAS5 expression vector. (A) Tumor volumes were calculated every 7 days. (B) Representative pictures of the tumors from each group at day 28. (C) The weight of tumors was determined at day 28. The levels of GAS5 (D) and miR-23a-3p (E) were detected by using RT-qPCR. (F) The protein level of PTEN was analyzed by using western blot analysis. ${ }^{\star *} \mathrm{P}<0.01$ compared with Vector. 